\title{
Sistema de control de Inventarios multicriterio difuso para repuestos
}

\author{
Fuzzy Multi-criteria Inventory Control System for Spare Parts \\ A. M. Paredes-Rodríguez ; ; V. L. Chud-Pantoja iD ; J. C. Osorio iD
}

\begin{abstract}
Resumen-El control de inventarios se ha convertido en uno de los temas más trabajados en la academia en colaboración con la industria debido a la alta representación que tienen los costos de almacenamiento de producto y los costos asociados a las ventas perdidas por no tener la disponibilidad del producto a la hora de la demanda. En este artículo se presenta una propuesta para el problema de control de inventarios de los ítems más representativos de un almacén de repuestos, donde se debe considerar más de un criterio. La metodología aplicada se basó inicialmente en la realización de una clasificación de los ítems mediante el uso del AHP difuso (FAHP), donde se tuvieron en cuenta importantes criterios relacionados con la caracterización del tipo de ítems, tales como el costo total y la criticidad, con esto se identificaron los grupos de ítems más representativos, después se realizó una segunda clasificación para identificar dentro de estos grupos los artículos críticos para el sistema de control. Posteriormente se realizó un análisis de demanda y el respectivo pronóstico para los ítems clasificados como tipo A. Finalmente se presenta la propuesta de aplicación de la política de control de inventario continua $(s, Q)$, teniendo en cuenta el análisis de las políticas utilizadas para los repuestos y se valida mediante un caso aplicado a un ingenio azucarero. Entre los principales resultados de la investigación se tiene la identificación de aquellas referencias más importantes para el ingenio considerando múltiples criterios, permitiendo establecer una política de inventario que minimiza el costo y cumple con un nivel de servicio previamente definido por la organización.
\end{abstract}

Palabras claves - AHP difuso, Clasificación ABC, Control de inventarios, Ingenios azucareros, Pronósticos, Repuestos.

Abstract - Inventory control has become one of the most worked topics in the academy in collaboration with the industry due to the high representation of product storage costs and the costs associated with lost sales due to not having product availability at the time of demand. This article presents a proposal for the inventory control problem of the most representative items of a spare parts warehouse, where more than one criterion must be considered. The methodology applied was initially based on the realization of a classification of the items through the use of diffuse AHP (FAHP), where important criteria related to the characterization of the type of items, such as total cost and criticality, were taken into account. with this, the most representative groups of items were identified, then a second

Este manuscrito fue enviado el 9 de agosto de 2019 y aceptado el 20 de diciembre de 2019. Este trabajo de investigación contó con el apoyo conjunto de la Universidad del Valle y la Corporación Universitaria Minuto de Dios.

Andrés M. Paredes es Ingeniero Industrial Egresado de la Universidad del Valle. Entre sus temas de interés se encuentra la optimización y simulación de cadenas de suministro. Docente Universidad Minuto de Dios seccional Valle. E-mail: andres.paredes@ correounivalle.edu.co classification was made to identify within these groups the critical articles for the control system. Subsequently, a demand analysis and the respective forecast for the items classified as type $A$ were carried out. Finally, the proposal for the application of the continuous inventory control policy $(s, Q)$ is presented, taking into account the analysis of the policies used for the spare parts and is validated by a case applied to a sugar mill. Among the main results of the investigation is the identification of those most important references for the mill considering multiple criteria, allowing to establish an inventory policy that minimizes the cost and meets a level of service previously defined by the organization.

Index Terms - Fuzzy AHP, Classification ABC, Inventory control, sugar mill, forecasting, spare parts.

\section{INTRODUCCIÓN}

$\mathrm{E}^{\mathrm{N}}$ la actualidad el entorno al cual se enfrentan las compañías genera que estas deban de tener unidades disponibles en su almacén para responder a la variabilidad e incertidumbre de la demanda del cliente; por tal motivo, el control de inventarios se ha convertido en uno de los temas más complejos y apasionantes de la logística ya que es un tema aplicable a cualquier tipo de industria [1]. Se debe aclarar que los motivos principales por los que surge la necesidad de mantenimiento de inventarios son las fluctuaciones aleatorias de la demanda y el tiempo de suministro o lead time.

Asimismo, la clasificación de productos de repuesto dentro de un inventario permite a las organizaciones definir políticas de inventario para aquellos artículos de mayor criticidad, no solo en términos de representación en ventas sino también considerando criterios cualitativos como tamaño, forma, impacto en la operación, entre otros. Por consiguiente, la valoración de la importancia de un producto a través de múltiples criterios es la principal ventaja del enfoque multicriterio comparado con la clasificación ABC Tradicional donde solo se tiene en cuenta el volumen de ventas de los artículos.

De esta manera, en este documento se propone una metodología multicriterio para establecer una política de inventarios de los ítems más representativos en un almacén de repuestos, que

Vivian L. Chud es Magister en Ingeniería Industrial de la Universidad del Valle. Profesora tiempo completo de Ingeniería Industrial, Universidad del Valle Sede Zarzal. E-mail: Vivian.chud@correounivalle.edu.co

Juan C. Osorio es Doctor en Ingeniería Industrial de la Universidad del Valle. E-mail: juan.osorio@correounivalle.edu.co. Profesor Titular, Escuela de Ingeniería Industrial, Universidad del Valle. 
pretende aportar a la función que tiene el control de inventario de piezas de repuesto, que busca ayudar al personal de mantenimiento a conservar los equipos en las condiciones óptimas de funcionamiento [2]. Dado que las piezas de repuesto no son productos intermedios o finales para ser vendidos a un cliente, existen aspectos únicos de estos ítems que deben tenerse en cuenta:

- Las políticas de mantenimiento y gestión de inventario de piezas de repuestos se establecen de acuerdo a la necesidad y criticidad de una pieza, la cual es necesaria para el adecuado funcionamiento de las máquinas y el correcto desarrollo del producto.

- Generalmente no se encuentra información confiable disponible necesaria para la predicción de tiempos de falla, sobre todo en el caso de los nuevos equipos.

- Los costos de este tipo de artículos son de gran importancia ya que generalmente influyen en la calidad, así como en la pérdida de producción, y suelen ser difíciles de cuantificar.

- La obsolescencia puede ser un problema, ya que las máquinas para las que las piezas de repuesto fueron diseñadas pueden quedar como obsoletas y ser sustituidas [2]

En esta investigación se propone el uso de la clasificación $\mathrm{ABC}$ multicriterio difusa (FAHP) debido a que los números difusos permiten cuantificar criterios cualitativos, los cuales no tienen una escala de medición precisa. Entre las aplicaciones en inventario de esta metodología se encuentra la investigación realizada por [3], quienes desarrollan una clasificación del inventario teniendo en cuenta criterios cuantitativos y cualitativos que son valorados a partir de la experiencia de los expertos. Igualmente, el diseño de un sistema de control de inventarios eficiente que permita a las organizaciones llevar un mayor control de aquellos artículos de mayor relevancia para aumentar el flujo de caja de la compañía, a través de la reducción de ventas pérdidas por productos agotados y la disminución en compra de materiales de baja rotación, también se establecen los pasos que debe tenerse en cuenta para artículos de repuestos.

Diferentes autores han abordado el control de inventarios para la gestión de repuestos [4], [5], [6] y [7].

Este artículo se encuentra distribuido de la siguiente forma: en la sección 2 se presenta una breve revisión sobre políticas de inventario de repuestos; en el inciso 3 se explica la metodología utilizada para el estudio y se termina exponiendo los principales resultados y conclusiones en los apartados 4 y 5 respectivamente.

\section{REVISIÓN DE LA LITERATURA}

Se analizaron 21 artículos relacionados con las políticas de inventarios. El resumen consolidado de estos se muestra en la Tabla I.

Entre los principales resultados se encuentra que el 60,9\% (14 documentos) utilizan una política de revisión continua. Entre las políticas de revisión continua más utilizadas se encuentra la política s, Q o R,Q (9 documentos) seguido de las políticas s,S (3 documentos) y S-1,S (3 documentos).

Un aspecto importante analizado es que la demanda de este tipo de ítem es errática o intermitente debido a que el cliente, que por lo general es el área de mantenimiento, no necesita continuamente un repuesto en específico, sino que solicita múltiples piezas de repuestos en distintos intervalos de tiempo.

TABLA I

RESUMEN De REvisión De LA Literatura Sobre Sistemas De INVENTARIO.

\begin{tabular}{|c|c|c|c|c|}
\hline Autores & Revisión & $\begin{array}{l}\text { Sistema de } \\
\text { inventario }\end{array}$ & Caso & Industria \\
\hline [8] & Continua & $\mathrm{s}, \mathrm{S}$ & Teórico & NA \\
\hline [9] & Continua & $\mathrm{s}, \mathrm{Q}$ & Teórico & NA \\
\hline [10] & Lote a lote & $(\mathrm{S}-1, \mathrm{~S})$ & Práctico & Petroquímica \\
\hline$[2]$ & $\begin{array}{l}\text { Continua, } \\
\text { periódica, y } \\
\text { modelos } \\
\text { basados en } \\
\text { sistemas de } \\
\text { colas } \\
\text { cíclicos }\end{array}$ & $\begin{array}{c}(\mathrm{S}, \mathrm{s}) ;(\mathrm{S}-1, \\
\mathrm{s})\end{array}$ & Práctico & $\begin{array}{l}\text { Aeroplanos, } \\
\text { automotriz, } \\
\text { aeroespacial }\end{array}$ \\
\hline [11] & Continua & $(\mathrm{s}, \mathrm{nQ})$ & Práctico & Petroquímica \\
\hline [12] & Continua & $\mathrm{s}, \mathrm{Q}$ & Teórico & NA \\
\hline [13] & Continua & $(\mathrm{S}-1, \mathrm{~S})$ & Revisión & Aviación \\
\hline [14] & Continua & EOQ & Teórico & No aplica \\
\hline [15] & Periódica & $(\mathrm{S}, \mathrm{Q})$ & Teórico & $\begin{array}{l}\text { Cualquier línea de } \\
\text { producción }\end{array}$ \\
\hline [4] & Periódica & $\begin{array}{l}(\mathrm{R}, \mathrm{S}) \mathrm{y} \\
(\mathrm{R}, \mathrm{s}, \mathrm{S})\end{array}$ & Práctico & $\begin{array}{l}\text { Manufacturera de } \\
\text { electrodomésticos } \\
\text { de línea blanca }\end{array}$ \\
\hline [16] & Periódica & $\begin{array}{l}\text { Modelo } \\
\text { EOQ } \\
(\mathrm{R}, \mathrm{s}, \mathrm{S})\end{array}$ & Práctico & Química \\
\hline [17] & $\begin{array}{l}\text { Continua- } \\
\text { Evaluación } \\
\text { Periódica }\end{array}$ & $\begin{array}{l}(\mathrm{R}, \mathrm{S}) \& \\
(\mathrm{~s}, \mathrm{~S})\end{array}$ & Teórico & $\begin{array}{l}\text { Refinería de } \\
\text { Petróleo }\end{array}$ \\
\hline [18] & Continua & $\begin{array}{l}(\mathrm{s}-1, \mathrm{~s}) \text { y } \\
(\mathrm{s}, \mathrm{Q})\end{array}$ & Práctico & Militar \\
\hline [19] & Continua & $(\mathrm{S}-1, \mathrm{~S})$ & Revisión & Sector Militar \\
\hline [20] & Continua & $\mathrm{s}, \mathrm{Q}$ & Práctico & Automotriz \\
\hline [21] & NA & NA & Práctico & Líneas aéreas \\
\hline [22] & Periódica & $(\mathrm{R}, \mathrm{S})$ & Teórico & $\begin{array}{c}\text { Compañía de } \\
\text { locomotoras } \\
\text { eléctricas }\end{array}$ \\
\hline$[6]$ & Continua & $\mathrm{s}, \mathrm{S}$ & Práctico & Energética \\
\hline [23] & NA & $\begin{array}{c}\text { NA - } \\
\text { clasificación } \\
\text { de } \\
\text { inventario }\end{array}$ & Teórico & Eléctrica \\
\hline
\end{tabular}

La complejidad en el control de inventario de repuestos se asocia a la cantidad de ítems que manejan los almacenes, por ejemplo, en la revisión se encontró que alrededor de una cuarta parte de los inventarios de las compañías que fueron utilizadas por los autores tenían más de 10.000 referencias en sus almacenes. Además, entre las empresas que más necesitan un 
control de inventario de repuestos se tiene la industria de aviación, seguido de la industria petroquímica y la industria automotriz y energética.

\section{METODOLOGÍA}

La metodología propuesta en este artículo para diseñar un sistema de control de inventarios multicriterio difuso, consta de tres fases generales (ver figura 1): La primera hace referencia a una clasificación $\mathrm{ABC}$ multicriterio difusa de los productos, donde se categoriza cada uno de los artículos del inventario según su nivel de importancia, considerando el juicio de expertos y los diferentes criterios requeridos para definir la categoría de cada producto; la segunda etapa consiste en la definición de un sistema de pronósticos para cada tipo de producto (A, B y C); por último, se tiene la definición de la política de inventario que mejor se adapte al comportamiento de cada ítem. A continuación, se presentarán los detalles de cada una de las fases.

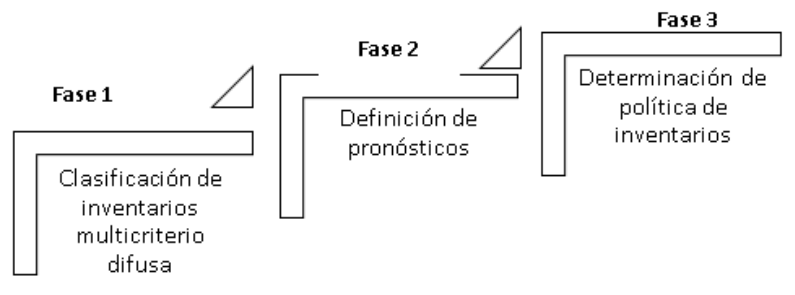

Fig 1. Pasos para el diseño de un sistema de control de inventarios multicriterio difuso

Fase 1. Clasificación de inventarios

En esta fase se consideran cuatro pasos para realizar una clasificación de inventario multicriterio considerando aspectos difusos, estos se presentan en la Fig 2.

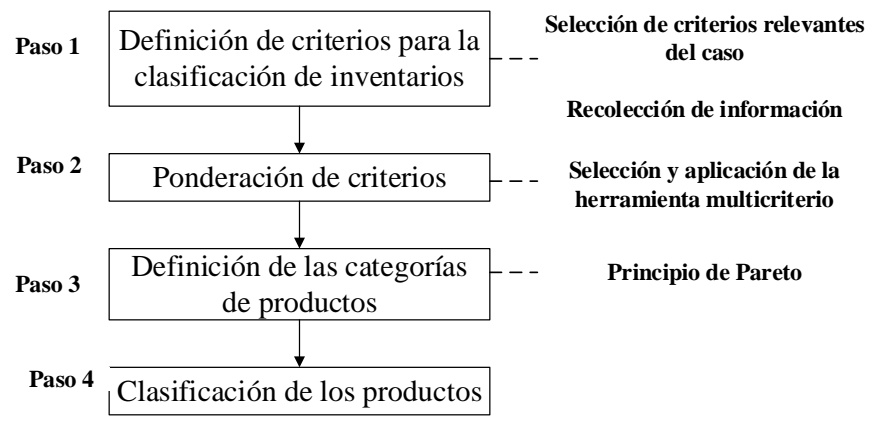

Fig 2. Metodología para la clasificación de inventarios multicriterio

\section{Paso 1. Definición de los criterios}

Una buena elección de los criterios garantiza una mayor efectividad en la clasificación del inventario; por ejemplo la clasificación $\mathrm{ABC}$ tradicional solo tiene en cuenta un criterio de volumen de ventas, lo que hace que esta metodología tenga una visión sesgada y deje a un lado otros criterios que pueden ser importantes para la organización y que están ligados directamente a la naturaleza propia de los ítems. Para definir los criterios a utilizar en este estudio, se realizó una revisión de la literatura sobre la clasificación ABC (La tabla II resume los aspectos más relevantes). El paso a seguir consiste en seleccionar aquellos criterios más importantes para las personas encargadas de tomar la decisión (en este grupo normalmente se incluye el responsable del almacén, los jefes de compra, producción, ventas, entre otros).

TABLA II

CRITERIOS Y HERRAMIENTAS MULTICRITERIO UTILIZADAS EN LA CLASIFICACIÓN DE INVENTARIOS

\begin{tabular}{|c|c|c|c|}
\hline "Herramienta & Criterios & Producto & Autor \\
\hline $\begin{array}{l}\text { AHP - TOPSIS } \\
\text { ABC }\end{array}$ & $\begin{array}{l}\text { Costo promedio de } \\
\text { Unidad } \\
\text { Lead time } \\
\text { Nivel de demanda } \\
\text { anual } \\
\text { Criticidad }\end{array}$ & Electrónicos & [23] \\
\hline $\begin{array}{l}\text { Clasificación de } \\
\text { Inventario } \\
\text { Multicriterio }\end{array}$ & $\begin{array}{l}\text { Volumen de ventas } \\
\text { Coeficiente de } \\
\text { variación de la } \\
\text { demanda } \\
\text { Número de órdenes } \\
\text { Duración } \\
\text { Ingreso bruto }\end{array}$ & $\begin{array}{l}\text { Fabricante } \\
\text { de queso }\end{array}$ & [24] \\
\hline $\begin{array}{l}\text { Método de } \\
\text { puntuación }\end{array}$ & $\begin{array}{l}\text { Valor } \\
\text { Lead time } \\
\text { Patrón de demanda }\end{array}$ & $\begin{array}{l}\text { Resistencias } \\
\text { eléctricas }\end{array}$ & [25] \\
\hline $\begin{array}{l}\text { Análisis ABC } \\
\text { Multicriterio } \\
\text { Método de } \\
\text { asignación lineal } \\
\text { difuso }\end{array}$ & & & [26] \\
\hline $\begin{array}{l}\text { Modelo de } \\
\text { clasificación } \\
\text { multicriterio } \\
\text { basado en reglas } \\
\text { difusas }\end{array}$ & $\begin{array}{l}\text { Valor de consumo } \\
\text { Precio unitario } \\
\text { Lead time } \\
\text { Criticidad } \\
\text { Similitud }\end{array}$ & $\begin{array}{l}\text { Diversas } \\
\text { industrias }\end{array}$ & [27] \\
\hline $\begin{array}{l}\text { Clasificación } \\
\text { multicriterio } \\
\text { jerárquica }\end{array}$ & $\begin{array}{l}\text { Fase del ciclo de } \\
\text { ventas } \\
\text { Tiempo de entrega a } \\
\text { clientes } \\
\text { Número de ordenes } \\
\text { Frecuencia de } \\
\text { demanda } \\
\text { Parte crítica } \\
\text { Parte del valor }\end{array}$ & Repuestos & [28] \\
\hline $\begin{array}{l}\text { Clasificación } \\
\text { ABC con AHP }\end{array}$ & $\begin{array}{l}\text { Fase del ciclo de } \\
\text { ventas } \\
\text { Lead time } \\
\text { Número de órdenes } \\
\text { Demanda } \\
\text { Frecuencia } \\
\text { Criticidad } \\
\text { Costo. }\end{array}$ & $\begin{array}{l}\text { Manufacture } \\
\text { ra de } \\
\text { electrodomés } \\
\text { ticos de línea } \\
\text { blanca }\end{array}$ & [4] \\
\hline $\begin{array}{l}\text { Clasificación } \\
\text { difusa } A B C\end{array}$ & $\begin{array}{l}\text { Valor } \\
\text { Criticidad } \\
\text { Frecuencia de } \\
\text { demanda }\end{array}$ & Repuestos & [14] \\
\hline AHP & $\begin{array}{l}\text { Demanda } \\
\text { Costo unitario } \\
\text { Sustituibilidad } \\
\text { Términos de pago }\end{array}$ & $\begin{array}{l}\text { Productos de } \\
\text { consumo } \\
\text { masivo }\end{array}$ & [29] \\
\hline
\end{tabular}

Entre las herramientas utilizadas para conseguir un óptimo control de inventario de piezas de repuesto se tiene el enfoque de optimización, la clasificación $\mathrm{ABC}$ tradicional o multicriterio y modelos analíticos desarrollados por los autores. 
Se encontró que, de acuerdo con la revisión, los criterios más importantes para el control de inventario de repuestos son: el nivel de servicio y el costo debido a que se debe establecer una política que permita disminuir al máximo el costo por almacenamiento, obsolescencia y pedido, a la vez que se responde efectivamente a la demanda del cliente, buscando evitar rupturas de inventarios de repuestos altamente críticos para el proceso.

\section{Recolección de los datos}

Una vez se encuentran definidos los criterios, se procede a levantar la información de estos para cada uno de los productos que se encuentran dentro del inventario. Esta etapa es fundamental, ya que la información que se va a utilizar en el análisis $\mathrm{ABC}$ debe ser confiable y fiel a la realidad. Como insumo general se necesita un análisis de las ventas (expresadas en términos de dinero y cantidad) y los otros datos que se deben recolectar, dependerán de los criterios inicialmente seleccionados.

\section{Paso 2. Ponderación de criterios}

Para realizar la ponderación o asignación de los pesos de los criterios, se puede utilizar una herramienta multicriterio que permita valorar los atributos propios de la naturaleza de los productos que se encuentran en inventario.

\section{Definición de la herramienta multicriterio}

El siguiente paso es elegir aquella herramienta multicriterio que mejor se adapte al problema en cuestión. En la actualidad existen un grupo amplio de metodologías de análisis multicriterio, la cual presentan ventajas y desventajas (en la tabla 3 se definen algunas herramientas utilizadas junto con sus propiedades características)

Se define que una buena herramienta a utilizar es AHP - difuso, dado que permite la valoración de criterios cuantitativos y cualitativos, y al trabajar con números difusos, se permite manejar la incertidumbre, imprecisión y subjetividad inherente a la decisión humana o al juicio de los expertos. A su vez, una herramienta de decisión AHP permite que se trabaje solo con los criterios de mayor relevancia para la investigación [29] debido a que, dependiendo del tipo de industria y la naturaleza del ítem, los criterios adquieren diferentes pesos.

TABLA III

PRINCIPALES HERRAMIENTAS MUlTiCRITERIO UtILIZADAS PARA CLASIFICACIÓN ABC

\begin{tabular}{|c|c|c|}
\hline $\begin{array}{l}\text { Herramienta } \\
\text { multicriterio }\end{array}$ & Ventajas & Artículos referentes \\
\hline $\begin{array}{l}\text { Proceso Analítico } \\
\text { Jerárquico (AHP) }\end{array}$ & $\begin{array}{l}\text { Establece una escala } \\
\text { de valoración para los } \\
\text { criterios (Escala Saaty) }\end{array}$ & [30]; [31]; [32]; [33] \\
\hline $\begin{array}{l}\text { TOPSIS (Técnica de } \\
\text { Ordenamiento de } \\
\text { preferencia por } \\
\text { similitud con la } \\
\text { solución ideal) }\end{array}$ & $\begin{array}{l}\text { Más que una } \\
\text { herramienta de } \\
\text { selección, es una } \\
\text { metodología para } \\
\text { ordenar las diferentes } \\
\text { alternativas a evaluar }\end{array}$ & [34] \\
\hline
\end{tabular}

\begin{tabular}{|c|c|c|}
\hline $\begin{array}{l}\text { Lógica difusa (Fuzzy } \\
\text { Logic) }\end{array}$ & $\begin{array}{l}\text { Permite valorar } \\
\text { criterios cualitativos a } \\
\text { través de la definición } \\
\text { de escalas subjetivas }\end{array}$ & [35] \\
\hline $\begin{array}{l}\text { Proceso analítico en } \\
\text { red (ANP) }\end{array}$ & $\begin{array}{l}\text { Se puede trabajar con } \\
\text { criterios que no tienen } \\
\text { que estar organizados } \\
\text { en forma de jerarquía }\end{array}$ & [36] \\
\hline $\begin{array}{l}\text { Despliegue de la } \\
\text { función de calidad } \\
\text { (QFD) }\end{array}$ & $\begin{array}{l}\text { Tiene en cuenta las } \\
\text { necesidades del cliente } \\
\text { y las características } \\
\text { propias de la } \\
\text { organización a la hora } \\
\text { de tomar la decisión }\end{array}$ & [37]; [38] \\
\hline AHP-Difuso & $\begin{array}{l}\text { Permite manejar la } \\
\text { incertidumbre de la } \\
\text { valoración de los } \\
\text { expertos a través de } \\
\text { una escala de Saaty } \\
\text { definida con números } \\
\text { difusos }\end{array}$ & [3]; [39] \\
\hline AHP-TOPSIS & $\begin{array}{l}\text { La valoración de } \\
\text { criterios se realiza } \\
\text { utilizando el método } \\
\text { tradicional de AHP y } \\
\text { la evaluación de los } \\
\text { ítems con TOPSIS } \\
\text { permite ordenarlos } \\
\text { según su importancia }\end{array}$ & [40] \\
\hline QFD-Difuso & $\begin{array}{l}\text { Permite tener en } \\
\text { cuenta escalas } \\
\text { subjetivas en la } \\
\text { valoración del QFD } \\
\text { original }\end{array}$ & [37]; [38] \\
\hline
\end{tabular}

\section{Asignación de los pesos a los criterios}

En esta fase se deben buscar expertos que tengan conocimiento sobre el inventario que va a ser clasificado, para que valoren los criterios en una matriz de comparaciones, donde se busca establecer aquellos atributos que poseen una mayor importancia para las partes interesadas. Para la valoración de los criterios se utiliza la escala de Saaty (para mayor información sobre esta escala, consulte [41]), la cual se utiliza en el proceso analítico jerárquico (AHP) para la comparación de criterios. Esta escala otorga un peso del 1 al 9, donde 1 se utiliza cuando los criterios comparados tienen el mismo grado de importancia y 9 cuando la relación del primer atributo es absolutamente más importante que el segundo.

Se debe tener en cuenta que, a la hora de la calificación de los criterios, se utilizan números difusos trapezoidales que tienen una función de pertenencia (A-1, A, A, A+1), donde A es la ponderación dada al criterio; por ejemplo, si se le da una calificación de 5 , este valor se representa como el número difuso $(4,5,5,6)$

Una vez todos los expertos han ponderado la matriz de criterios, se procede a realizar un promedio ponderado de las calificaciones dependiendo de la importancia de cada evaluador y seguido de esto se procede a defuzificar los valores a través de (1): 


$$
\text { Valor defuzificado }=\frac{a+2(b+c)+d}{6}
$$

\section{Teniendo en cuenta un número difuso de la forma $(a, b, c, d)$}

Con la matriz defuzificada se calcula la prioridad de cada uno de los criterios y luego se procede a realizar un análisis de consistencia (para revisar este paso se puede consultar [42]) para verificar uniformidad en los juicios establecidos en la matriz.

\section{Paso 3. Definición de la categoría de productos}

En algunas ocasiones, debido al volumen de artículos que maneje la empresa dentro de su inventario, se hace necesario realizar una clasificación por categoría de producto, donde los ítems pertenecientes a cada conjunto deben compartir una característica similar (uso, costo, tipo de almacenamiento, entre otros).

Una vez se han establecido los pesos de los criterios, se debe valorar cada grupo respecto a los atributos seleccionados (los cuales pueden ser tomados de aquellos criterios encontrados en la revisión de la literatura y que se encuentra expuestos en la tabla 2). Se debe tener en cuenta que dependiendo del criterio se tienen distintas escalas de valoración. Por ejemplo, las ventas se miden en unidades de dinero (pesos, dólares, euros, entre otros), mientras que otros criterios como el tiempo de entrega se miden en horas, días e incluso semanas.

Teniendo en cuenta la valoración de cada categoría se procede a convertir las calificaciones en número difusos trapezoidales, de la forma en que se mencionó en el paso 2. Luego de esto se multiplica la matriz de valoraciones con la matriz de pesos de criterios para de esta forma obtener la calificación final de cada ítem.

Por último, se organizan las calificaciones obtenidas en orden descendente y se procede a calcular el porcentaje de participación respecto al total. Teniendo en cuenta el porcentaje de participación acumulado se realiza la clasificación ABC. Los grupos que representan el $80 \%$ de la participación acumulada se clasifican como tipo A; asimismo, el siguiente $15 \%$ se categorizan como conjuntos tipo B y el último $5 \%$ como categorías tipo C.

\section{Paso 4. Clasificación de los productos}

Las categorías de producto que fueron calificadas como tipo A, son desglosadas y ahora el análisis elaborado en el paso 3 se realiza por ítem, buscando identificar aquellos artículos más críticos para la organización. Es necesario tener en cuenta que para la clasificación por producto se utiliza el mismo principio de Pareto utilizado en el inciso anterior (80\% productos tipo A, $15 \%$ artículos tipo B y $5 \%$ ítems tipo C).

\section{Fase 2. Definición de pronósticos}

Una vez se han clasificado los productos (A, B y C) se procede a analizar el comportamiento de cada uno de ellos para identificar un patrón o tendencia que se ajuste con uno de los sistemas de pronósticos. Los pasos que se deben seguir para seleccionar el mejor modelo de pronóstico son los siguientes:

\section{Análisis de demanda}

El dato de entrada más importante para seleccionar un adecuado sistema de pronósticos es el histórico de la demanda de un ítem. En esta etapa es importante validar la confiabilidad de los datos encontrados, evitando que algún registro inadecuado pueda alterar el patrón real de demanda del producto. Asimismo, es necesario proponer una estrategia para que los puntos atípicos de demanda (llamados comúnmente outliers) no alteren el pronóstico de los datos; las dos estrategias más utilizadas para esto son la eliminación de los outliers o el reemplazo de estos puntos atípicos por el promedio de los datos.

Para identificar cuál es el patrón que mejor se adapta a cada artículo, se utiliza frecuentemente el coeficiente de variación de los respectivos datos de demanda, se calcula según (2). Cuando este coeficiente tiene un valor mayor a 1, significa que el patrón de demanda es errático o estacionario. Si la variación es cercana a cero representa un comportamiento perpetuo o uniforme.

$$
C V=\frac{\sigma}{X}
$$

\section{Elección de sistemas de pronósticos}

Una vez se identifique los patrones de demanda de aquellos artículos de mayor criticidad para la organización (normalmente llamados productos tipo A), se procede a analizar qué sistema de pronóstico se adapta mejor a cada ítem, dependiendo de su comportamiento histórico. [1] establece una relación del sistema de pronóstico recomendado según el patrón de demanda (ver tabla IV)

TABLA IV

SELECCIÓN DE PRONÓSTICO RECOMENDADO DEPENDIENDO

\begin{tabular}{|c|c|}
\hline Patrón de demanda & $\begin{array}{c}\text { Sistema de pronóstico } \\
\text { recomendado }\end{array}$ \\
\hline Perpetuo o uniforme & $\begin{array}{l}\text { Promedio móvil o suavización } \\
\text { exponencial simple }\end{array}$ \\
\hline $\begin{array}{c}\text { Con tendencia creciente o } \\
\text { decreciente }\end{array}$ & Suavización exponencial doble \\
\hline Estacional o periódica & Modelos periódicos de Winters \\
\hline Demanda altamente correlacionadas & $\begin{array}{l}\text { Métodos integrados de promedio } \\
\text { móviles auto-regresivos (ARIMA) }\end{array}$ \\
\hline $\begin{array}{c}\text { Errática (ítems clase A de bajo } \\
\text { movimiento) }\end{array}$ & $\begin{array}{c}\text { Pronóstico combinado de tiempo } \\
\text { entre la ocurrencia de demanda } \\
\text { consecutivas y la magnitud de } \\
\text { transacciones }\end{array}$ \\
\hline
\end{tabular}
DEL PATRÓN DE DEMANDA [1]

Es necesario tener en cuenta, que a pesar que hay unos sistemas recomendados según el comportamiento de la demanda, la eficiencia de un sistema de pronóstico se mide a través del cálculo del error del método utilizado, donde se selecciona aquel sistema de pronóstico que obtengan el menor error. Las dos medidas que se usan en la actualidad son el Error Medio Absoluto (MAD) y el Error Cuadrático Medio (ECM).

\section{Definición de parámetros base para la política de inventarios}


Con el sistema de pronóstico seleccionado, se procede a simular el comportamiento futuro de la demanda, con el objetivo de obtener información que sirva de insumo para la política de inventarios. Dependiendo de la estrategia de inventario a utilizar, el sistema de pronóstico ofrece los siguientes parámetros fundamentales para la definición de la política

Valor de demanda futura: Es la proyección de demanda que ofrece el sistema de pronóstico. Se utiliza frecuentemente para estimar la demanda promedio que ocurrirá cuando el producto viene en tránsito desde el proveedor (Lead time)

Desviación estándar de la demanda: Representa la variabilidad de los datos de demanda. Se utiliza para determinar los inventarios de seguridad, el cual es el producto que se tiene para cumplirle al cliente en caso de una variación inesperada de la demanda.

\section{Fase 3. Determinación de políticas de inventario}

Una de las tareas más complejas para las empresas industriales y comerciales es la administración y control de inventarios [43]. En la actualidad existen diversas políticas de inventario, las cuales se pueden clasificar dependiendo al patrón de demanda y variabilidad de los tiempos de reposición [1]. A continuación, se establece el paso a paso que se debe seguir para la determinación de las políticas

\section{Definición teórica de las políticas de control de inventario}

La clasificación más general de las políticas de inventario se conforma por sistemas de revisión continua y periódica. A continuación, se presenta una breve explicación sobre las políticas más utilizadas

\section{Política de revisión continua $s, Q$}

Este sistema consiste en revisar el inventario cada vez que se realice una transacción y solicitar una cantidad de pedido (Q) cuando el inventario efectivo se encuentre por debajo de un punto de reorden (s).

\section{Política de revisión continua $s, S$}

Esta política consiste en revisar el inventario cada vez que se realice una transacción y solicitar una cantidad equivalente a la diferencia entre un inventario máximo (S) y el inventario efectivo. El momento en que se realiza la solicitud es cuando el inventario efectivo se encuentre por debajo del punto de reorden (s).

\section{Política de revisión periódica $R, S$}

En este sistema la revisión del inventario se realiza cada $\mathrm{R}$ unidades de tiempo y se solicita una cantidad equivalente a la diferencia entre el inventario máximo (S) y el inventario efectivo.

Política $R, s, S$
Es una política de inventarios híbrida donde se el inventario se revisa cada cierto intervalo de tiempo $(\mathrm{R})$ y se solicita producto, solo sí el inventario efectivo se encuentra por debajo del punto de reorden (s). La cantidad a solicitar es la diferencia entre el inventario máximo (S) y el inventario efectivo.

Definición de los datos requeridos para la política de inventarios

Debido a la importancia que tienen los repuestos en la operación del ingenio, se decide utilizar una política de revisión continúa s, Q. A continuación se establece el paso a paso a seguir

\section{Determinación de la cantidad a pedir $(Q)$}

El primer paso que se debe realizar es determinar la cantidad Q a solicitar. Normalmente se utiliza la fórmula del cálculo del Punto de pedido económico (EOQ), ver en (3).

$$
Q=\sqrt{\frac{2 A D}{v r}} \quad E c(3)
$$

Donde

$\mathrm{A}=$ Costo de realizar un pedido

$\mathrm{D}=$ Demanda anual pronosticada

$\mathrm{V}=$ Valor del producto

$\mathrm{R}=$ Tasa del costo de mantenimiento de inventario

\section{Definición del inventario de seguridad (IS)}

El siguiente paso es calcular el inventario de seguridad, tal y como lo muestra en (4). Para poder obtener este valor se debe tener la desviación estándar de la demanda pronosticada $(\sigma 1)$, el valor de $\mathrm{k}$ (el cual depende del nivel de servicio que se espera ofrecer al cliente final) y el lead time (L).

$$
\text { Inventario Seguridad }=k \sigma 1 \sqrt{L}
$$

\section{Cálculo del punto de reorden (s)}

Una vez se obtiene el inventario de seguridad, se procede a hallar el punto de reorden, el cual es la suma del inventario de seguridad con la demanda pronosticada para cubrir el lead time (XL) véase (5).

$$
S=X L+I S
$$

\section{RESULTADOS}

La metodología se validó mediante la aplicación en un ingenio azucarero, el cual se tomó como caso de estudio. En la actualidad el ingenio azucarero maneja aproximadamente 56.300 referencias de códigos representados en números de ocho dígitos, en donde cada uno describe un SKU con sus características específicas. Todos los ítems están clasificados en grupos, los que a su vez están divididos en subgrupos, esto contribuye a la fácil caracterización, agrupación y almacenamiento de los materiales en las mejores condiciones durante su estadía en el almacén. 
Los artículos se organizan dentro del almacén en grupos y subgrupos, por ejemplo, el grupo "TORNILLERIA", tiene los subgrupos de acero inoxidable, acero RF, acero galvanizado, etc. En cuanto al sistema de gestión de inventarios, el almacén no cuenta con cantidades de punto de re-orden de los artículos, ni niveles de inventario máximo o mínimo a almacenar. El sistema de inventarios consiste en almacenar las cantidades que requieren cada uno de los usuarios hasta el momento en que se necesite. El almacén es el encargado de darle el mejor manejo de los artículos solicitados con el fin de no retrasar las actividades principales del ingenio.

Debido a que se maneja gran volumen de códigos a ser controlados, semanalmente se realiza una inspección física de un grupo seleccionado de artículos, para verificar que las unidades que aparecen dentro del sistema corresponden con las unidades físicas existentes en el almacén. En ese sentido, se puede decir que el principal problema del ingenio es la carencia de una política de inventarios que le permita tener un control sobre los ítems más representativos de su almacén, y a su vez evitar altos costos de almacenamiento y/o ruptura de inventario.

Para resolver esta problemática, inicialmente se realizó una clasificación de los ítems obsoletos con ayuda de un experto de la empresa, donde se tuvo como resultado que solo 5000 de las 56.300 referencias son utilizadas actualmente en cada uno de los procesos de producción necesarios para el óptimo desempeño del Ingenio. Estas 5.000 referencias se encuentran divididas en 71 grupos diferentes dependiendo de las características propias de cada ítem. Una vez realizado este filtro, aplicó la clasificación ABC AHP-difuso con el objetivo de trabajar con el grupo de repuestos más representativo de los almacenados en esa bodega.

Para el caso de estudio se realizaron dos clasificaciones ABC AHP difuso: la primera para establecer los grupos de ítems más representativos en el inventario y la segunda para identificar dentro de estos grupos los artículos críticos para el sistema de control. Para la clasificación inicial se tuvieran en cuenta los atributos de costo total y criticidad; por el contrario, para la segunda categorización se utilizaron los criterios de costo total y rotación. Una vez aplicada la clasificación ABC presentada en la metodología se obtuvieron los siguientes ítems críticos: hierro corrugado de 5/8", 3 wa-53 juego de casquetes para eje de 6", ángulo de hierro de 3/8" x 2.1/2" x $6 \mathrm{mt}$, tubo de acero de 2 " shedule 10 , tubo cuadrado de 1 1/2" e: 1/16", en ac, lámina metaldek 2" calibre $22(0,75 \mathrm{~mm})$, tubos de hierro negro de $1.1 / 4$ " x 6 m y lamina inoxidable de $1 / 8$ " x 5" x 20 ".

Después de que se obtuvieron los 8 ítems más críticos del almacén de repuestos del ingenio se utilizó la demanda mensual histórica de las 8 referencias para calcular el coeficiente de variación de cada artículo y conocer el tipo de comportamiento, para proponer alguna herramienta de análisis de demanda que más se ajustara con el pronóstico.

Debido a que la demanda errática se caracteriza por tener periodos donde la demanda es igual a cero (0) unidades, es conveniente pronosticar las demandas futuras mediante el método Croston puesto que es la mejor estrategia para pronosticar artículos de demanda errática o intermitente [1]. En la tabla 3 que se presentan los resultados del pronóstico y los indicadores MAD y ECM, para 8 ítems clasificados como tipo A. En la tabla V se puede observar como el hierro corrugado de $5 / 8$ " es el que tiene un pronóstico de demanda mayor, sin embargo, su variación es alta debido al alto índice de error que posee (MAD igual a 71,22); por el contario el tubo de acero 2 ', tiene una demanda pequeña y muy estable (MAD igual a 3,17).

TABLA V

RESUMEN DE LOS RESULTADOS OBTENIDOS A PARTIR DEL PRONÓSTICO DE LOS ARTÍCULOS CLASE A MEDIANTE EL MÉTODO CROSTON

\begin{tabular}{|c|c|c|c|c|c|}
\hline & Ítem & $\begin{array}{c}\text { Periodo para } \\
\text { el cual se } \\
\text { pronostica }\end{array}$ & $\begin{array}{c}\text { Pronóstico } \\
\text { en Tiempo } \\
\text { Real }\end{array}$ & MAD & ECM \\
\hline 1 & $\begin{array}{c}\text { Hierro corrugado de } \\
5 / 8 "\end{array}$ & 27 & 67 & 71,22 & 10709,00 \\
\hline 2 & $\begin{array}{c}\text { juego de casquetes } \\
\text { de } 6,\end{array}$ & 27 & 18 & 13,19 & 509,96 \\
\hline 3 & $\begin{array}{c}\text { ángulo de hierro de } \\
3 / 8^{\prime},\end{array}$ & 27 & 12 & 29,18 & 3136,37 \\
\hline 4 & tubo de acero de 2 " & 27 & 4 & 3,17 & 12,24 \\
\hline 5 & $\begin{array}{l}\text { tubo cuadrado de } 1 \\
\qquad \frac{1}{2},\end{array}$ & 27 & 6 & 5,89 & 37,87 \\
\hline 6 & $\begin{array}{l}\text { lámina de metadelk } \\
2,,\end{array}$ & 27 & 16 & 22,32 & 500,99 \\
\hline 7 & $\begin{array}{l}\text { tubos de hierro } \\
\text { negro de } 1 \frac{1}{4},\end{array}$ & 27 & 15 & 15,20 & 262,19 \\
\hline 8 & $\begin{array}{c}\text { Lamina inoxidable } \\
\text { de } 1 / 8 " \times 5 "\end{array}$ & 27 & 1 & 0,99 & 1,19 \\
\hline
\end{tabular}

Para el presente análisis se evaluó la política de control de inventario s, Q para los 8 artículos pertenecientes a la clase A obtenidos mediante la clasificación ABC AHP difuso realizada anteriormente.

Para realizar el análisis se utilizó primeramente el error cuadrático medio (ECM) de cada artículo, y que fue obtenido a partir de los pronósticos hechos por medio del método Croston. Hallando la raíz cuadrada del ECM se obtuvo la desviación estándar de los errores del pronóstico $(\sigma 1)$. Para obtener el valor de la demanda mensual correspondiente al estudio, se utilizó el pronóstico de demanda calculado para cada uno de los ítems clase A.

De acuerdo con la política de la compañía, el 2,7\% de este costo es el valor utilizado como costo de ordenar para cada uno de los artículos evaluados en el presente estudio. A pesar de que este será el costo de ordenar que se va a utilizar para lo que haga falta, se recomienda una forma más práctica y exacta de calcular para que los encargados del almacén de repuestos del ingenio conozcan el verdadero costo de ordenar de cada artículo. Para el cálculo del respectivo costo se debería llevar un registro del costo por fletes, impuestos, del transporte de la orden, por preparar los formatos de las órdenes, de correo (fax, EDI, etc.), de llamadas telefónicas, de autorización del pedido, de recepción e inspección, de manejo de las facturas del proveedor, entre otros relacionados con el procesamiento de la orden. [1].

La tasa del costo de mantener el inventario (r) se determinó mediante una encuesta realizada a los encargados del inventario de repuesto en el almacén. El encargado brindó información 
sobre el valor de $\mathrm{r}$ para cada artículo como se muestra a continuación en la tabla VI.

$$
\text { TABLA VI }
$$

TAsa De Costo De Mantener INVENTARio De Los Ítems Clase A Del ALMACÉN DE REPUESTOS DEL INGENIO

\begin{tabular}{cc}
\hline \hline Ítem & Tasa de costo mantenimiento \\
\hline Hierro corrugado de 5/8" & $20 \%$ \\
juego de casquetes de 6" & $23 \%$ \\
ángulo de hierro de 3/8" & $20 \%$ \\
tubo de acero de 2" & $20 \%$ \\
tubo cuadrado de 1 1/2", & $21 \%$ \\
lámina de metadelk 2" & $22 \%$ \\
tubos de hierro negro de 1 1/4" & $20 \%$ \\
Lamina inoxidable de 1/8" x 5" & $22 \%$ \\
\hline \hline
\end{tabular}

El nivel de servicio al cliente es de $95 \%$, por esto se decidió manejar un nivel P1 del mismo valor, es decir, un nivel de servicio al cliente expresado en el número de veces que haya faltantes sin importar la cantidad de los mismo, debido a que al estar evaluando repuestos es importante tener en cuenta la criticidad de los mismos y se debe considerar que con una sola pieza que falte, la producción se pararía ocasionando diversos retrasos y altos costos.

Por último, se debió tomar en consideración un costo de faltantes el cual en este caso será B1 ya que, como se dijo anteriormente, los faltantes son representados por las veces en las que falta una pieza, más que por la cantidad de faltantes que se presente. Debido a que el estudio investigativo se está realizando en un almacén de repuestos, no habría un margen de utilidad que percibir por cada unidad faltante, pero ese faltante si se ve expresado en el costo asociado al detenimiento de la producción por suspender el uso de una máquina. Este costo debe determinarse a partir de la criticidad de determinada máquina, así como de la cantidad de máquinas paralelas que se tenga en la empresa. Se debe tener en cuenta que un componente del costo total relevante, como lo es el costo de faltante, se está buscando cuantificar y por ende para el ejercicio simplemente se asumió un valor constante. La política de inventarios para cada ítem se presenta en la tabla VII.

TABLA VII

POLÍTICA DE INVENTARIOS PARA ÍTEMS CLASE A

\begin{tabular}{|c|c|c|}
\hline Ítem & $\mathrm{S}$ & Q \\
\hline $\begin{array}{l}\text { Hierro corrugado de } \\
5 / 8 "\end{array}$ & 78 & 73 \\
\hline $\begin{array}{c}\text { juego de casquetes de } \\
6,\end{array}$ & 27 & 391 \\
\hline $\begin{array}{c}\text { ángulo de hierro de } \\
3 / 8 "\end{array}$ & 62 & 3835 \\
\hline tubo de acero de $2 "$ & 4 & 528 \\
\hline $\begin{array}{l}\text { tubo cuadrado de } 1 \\
\frac{1 / 2}{}, "\end{array}$ & 8 & 4131 \\
\hline $\begin{array}{c}\text { lámina de metadelk } \\
\qquad,\end{array}$ & 26 & 2968 \\
\hline $\begin{array}{l}\text { tubos de hierro negro } \\
\text { de } 1 \frac{1 / 4^{\prime}}{}\end{array}$ & 20 & 3022 \\
\hline $\begin{array}{c}\text { Lamina inoxidable de } \\
1 / 8^{\prime \prime} \times 5 "\end{array}$ & 1 & 400 \\
\hline
\end{tabular}

\section{CONCLUSIONES}

Se puede concluir que en la actualidad el almacén de repuestos del ingenio no cuenta con una política establecida de inventario, lo cual impide a los diferentes encargados de ésta sección conocer con exactitud cuántas unidades de cada SKU se encuentran disponibles. Así mismo, el no tener una visión clara de los ítems más representativos dentro del almacén de repuestos genera que se tengan una gran cantidad de productos de baja rotación y obsoletos.

La realización de una clasificación ABC AHP difuso permitió que se obtuvieran aquellos grupos de ítems de repuestos que son más críticos para las operaciones del Ingenio. Finalmente se trabajó con los 8 artículos más relevantes: el hierro corrugado, el juego de casquetes de 6', el ángulo de hierro de $3 / 8$ ', el tubo de acero de 2 ", el tubo cuadrado de $1 \frac{1}{2}$ ", la lámina de metadelk 2" y los tubos de hierro negro de $1 \frac{1 / 4}{4}$ " y la lámina inoxidable de 1/8" x 5". Estos artículos tienen un patrón de demanda errático debido a su naturaleza intermitente, por lo tanto, fue necesario que su demanda sea pronosticada por el método de Croston.

La realización de los pronósticos de demanda de los artículos clase A pertenecientes al almacén de repuesto del Ingenio es una actividad que permite tener un seguimiento de los repuestos más críticos para la realización de las diversas tareas de la empresa. De comenzar a realizar esta actividad en el Ingenio, se podría llegar a mantener el inventario justo y suficiente para prevenir un futuro faltante. Así mismo la información conseguida mediante los pronósticos, serán de gran ayuda para la realización, evaluación y selección de las diferentes políticas de control de inventario.

\section{REFERENCIAS}

C. J. Vidal, Fundamentos de control y gestión de inventarios. 2010.

2] W.J. Kennedy, J.W. Patterson and L.D. Fredendall, " An overview of recent literature on spare parts inventories", International journal of production economics, pp 201-215, 1996. DOI: 10.1016/S09255273(01)00174-8

[3] C. W. Chu, G. S. Liang, and C. T. Liao, "Controlling inventory by combining $\mathrm{ABC}$ analysis and fuzzy classification," Comput. Ind. Eng., vol. 55, no. 4, pp. 841-851, 2008. DOI: 10.1016/j.cie.2008.03.006

[4] A. Bacchetti and N. Saccani, "Spare parts classification and demand forecasting for stock control: Investigating the gap between research and practice," Omega, vol. 40, no. 6, pp. 722-737, Dec. 2012. DOI: 10.1016/j.omega.2011.06.008

[5] R. J. I. Basten and G. J. van Houtum, "System-oriented inventory models for spare parts," Surv. Oper. Res. Manag. Sci., vol. 19, no. 1, pp. 34-55, Jan. 2014. DOI: 10.1016/j.sorms.2014.05.002

[6] M. Guajardo, M. Rönnqvist, A. M. Halvorsen, and S. I. Kallevik, "Inventory management of spare parts in an energy company," J. Oper. Res. Soc., vol. 66, no. 2, pp. 331-341, 2015. DOI: 10.1057/jors.2014.8

[7] O. Bounou, A. El Barkany, and A. El Biyaali, "Inventory Models for Spare Parts Management: A Review," Int. J. Eng. Res. Africa, vol. 28, pp. 182-198, 2017.

[8] A.B.M.Z Kabir and A.S. Al-Olayan, " A stocking policy for spare part provisioning under age based preventive replacement". European Journal of Operation Research, Vol. 90, pp. 71-181, 2002.

[9] T. Shibuya, T. Dohi and S. Osaki, "Optimal continuous review policies for spare part provisioning with random lead times", International journal of productions economics; pp. 257-271, 1998. DOI: $10.1016 /$ S0925-5273(98)00065-6

[10] R. Dekker, M.J. Kleijn and P.J. De Rooij, "A spare parts stocking policy based on equipment criticality". International. Journal of Production Economics, pp. 69-77, 1998.

[11] E. Porras and R. Dekker, "An inventory control system for spare parts at a refinery: An empirical comparison of different re-order point methods", European Journal of Operations Research, pp 101-132, 2008. DOI: $10.1016 /$ j.ejor.2006.11.008

[12] T. Jin and H. Liao, "Spare parts inventory control considering stochastic growth of an installed base"; Computers \& Industrial 
Engineering, pp. 452-460, 2009. DOI: 10.1016/j.cie.2008.07.002

[13] M.P. Gato, M.C Carboneras, E. Babiloni and E. Guijarro, " Revisión de modelos de gestión de inventarios para repuestos reparables", 4 th International Conference on Industrial Engineering and Industrial Management, pp. 1329-1335, 2010.

[14] M. Bošnjaković, "MULTICRITERIA INVENTORY MODEL FOR SPARE PARTS,” pp. 499-504. DOI: 10.2139/ssrn.1362575

[15] Y. Song and Y. Wang, "Periodic review inventory systems with fixed order cost and uniform random yield", European Journal of Operational Research, 106-117, 2017. DOI: 10.1016/j.ejor.2016.07.005

[16] K. Velásquez, "Propuesta de un modelo de Inventario Para el Almacén de Repuestos de Mantenimiento en Clariant (Colombia) S.A", Tesis pregrado, Universidad Católica de Colombia.

[17] S. Panagiotidou, "Joint optimization of spare parts ordering and maintenance policies for multiple identical items subject to silent failures", European Journal of Operational Research, Vol 235, pp. 300-314, 2013.DOI: 10.1016/j.ejor.2013.10.065

[18] R. Minzhi, L. Yi and L. Hua, "Configuration model of partial repairable spares under batch ordering policy based on inventory state". Chinese Journal of Aeronautics, pp. 558-567, 2014. DOI: 10.1016/j.cja.2014.04.021

[19] R. J. I. Basten and G.J. van Houtum, "System-oriented inventory models for spare parts". Surveys in Operations Research and Management Science, pp., 34-55, 2014. DOI: 10.1016/j.sorms.2014.05.002

[20] J.R. Do Rego and M.A. Do Mesquita., "Demand forecasting and inventory control: A simulation study on automotive spare parts". International Journal of Production Economics, pp. 1-16, 2015. DOI: 10.1016/j.ijpe.2014.11.009

[21] J. Gu., G. Zhang and K.W Li, "Efficient aircraft spare parts inventory management under demand uncertainty". Journal of Air Transport Management, Vol 42, pp. 101-109, 2015. DOI: 10.1016/j.jairtraman.2014.09.006

[22] Y. Jiang,, M. Chen and D. Zhou, "Joint optimization of preventive maintenance and inventory policies for multi-unit systems subject to deteriorating spare part inventory"; Journal of manufacturing systems, pp. 191-205, 2015. DOI: 10.1016/j.jmsy.2015.01.002

[23] F. Arikan and S. Citak, "Multiple Criteria Inventory Classification in an Electronics Firm," Int. J. Inf. Technol. Decis. Mak., vol. 16, no. 02, pp. 315-331, 2017. DOI: 10.1142/S0219622017500018

[24] L. Yang, H. Li, J. F. Campbell, and D. C. Sweeney, "Integrated multiperiod dynamic inventory classification and control," Int. J. Prod. Econ., vol. 189, no. November 2016, pp. 86-96, 2017. DOI: 10.1016/j.ijpe.2017.04.010

[25] F. Lolli, A. Ishizaka, R. Gamberini, and B. Rimini, "A multicriteria framework for inventory classification and control with application to intermitten demand,"J Multi-Crit Decis Anal, vol. 1, no. 11, 2017. DOI: $10.1002 /$ mcda. 1620

[26] A. Baykasollu, K. Subulan, and F. S. Karaslan, "A new fuzzy linear assignment method for multi-attribute decision making with an application to spare parts inventory classification," Appl. Soft Comput. J., vol. 42, pp. 1-17, 2016. DOI: 10.1016/j.asoc.2016.01.031

[27] S. . Sarmah and U. . Moharana, "Multicriteria classification of spare parts inventories - a web based approach," J. Qual. Maint. Eng., vol. 21, no. 4, pp. 456-477, 2015. DOI: 10.1108/JQME-04-2012-0017

[28] A. Bacchetti, F. Plebani, N. Saccani, and A. A. Syntetos, "Empirically-driven hierarchical classification of stock keeping units," Int. J. Prod. Econ., vol. 143, no. 2, pp. 263-274, 2013. DOI: 10.1016/j.ijpe.2012.06.010

[29] F. Çebi, C. Kahraman, and B. Bolat, "A multiattribute ABC classification model using fuzzy AHP," 40th Int. Conf. Comput. Ind. Eng. Soft Comput. Tech. Adv. Manuf. Serv. Syst. CIE40 2010, 2010. DOI: $10.1109 /$ ICCIE. 2010.5668233

[30] B. E. Flores, D. L. Olson, and V. K. Dorai, "MANAGEMENT INVENTORY OF MULTICRITERIA CLASSIFICATION," Math. Comput. Model., vol. 16, no. 12, pp. 71-82, 1992.

[31] Y. Chen, K. W. Li, and S. Liu, "A Comparative Study on Multicriteria ABC Analysis in Inventory Management," 2008 IEEE Int. Conf. Syst. Man Cybern., pp. 280-285, 2008. DOI: 10.1109/ICSMC.2008.4811802

[32]
[33] K. Balaji and V. S. S. Kumar, "Multicriteria Inventory ABC Classification in an Automobile Rubber Components Manufacturing Industry," Procedia CIRP, vol. 17, pp. 463-468, 2014. DOI: 10.1016/j.procir.2014.02.044

[34] A. Bhattacharya, B. Sarkar, and S. Mukherjee, "Distance-based consensus method for ABC analysis," Int. J. Prod. Res., vol. 45, no. 15, pp. 3405-3420, 2007 DOI: 10.1080/00207540600847145

[35] A. Baykasoglu, K. Subulan, and F. S. Karaslan, "A new fuzzy linear assignment method for multi-attribute decision making with an application to spare parts inventory classification," Appl. Soft Comput., vol. 42, pp. 1-17, 2016. DOI: 10.1016/j.asoc.2016.01.031

[36] F. De Felice, D. Falcone, A. Forcina, A. Petrillo, and A. Silvestri, Inventory management using both quantitative and qualitative criteria in manufacturing system, vol. 47, no. 3. IFAC, 2014. DOI: 10.3182/20140824-6-ZA-1003.02279

[37] J. C. Osorio, D. C. Arango, and C. E. Ruales, "Selección de proveedores usando el despliegue de la función de calidad difusa," Rev. EIA, no. 15, pp. 73-83, 2011.

[38] J. C. Osorio, D. F. Manotas, and L. Rivera, "Priorización de Riesgos Operacionales para un Proveedor de Tercera Parte Logística - 3PL," Inf. tecnológica, vol. 28, no. 4, pp. 135-144, 2017.

[39] O. Cakir and M. S. Canbolat, "A web-based decision support system for multi-criteria inventory classification using fuzzy AHP methodology," vol. 35, pp. 1367-1378, 2008.

[40] J. C. Osorio and D. F. Manotas, "AHP Topsis para la selección de proveedores considerando el riesgo asociado a la calidad," Espacios, vol. 39 , no. 16,2018

[41] I. Basak and T. Saaty, "Group Decision Making using the Analytic Hierarchy Process," Math. Comput. Model., vol. 17, no. 415, pp. 101-109, 1993. DOI: 10.1016/0895-7177(93)90179-3

[42] K. Kułakowski, "Notes on order preservation and consistency in AHP," vol. 245, pp. 333-337, 2015. DOI: 10.1016/j.ejor.2015.03.010

[43] C. J. Vidal, J. C. Londoño, and F. Contreras, "Aplicación de Modelos de Inventarios en una Cadena de Abastecimiento de Productos de Consumo Masivo con una Bodega y N Puntos de Venta," Ing. y Compet., vol. 6, no. 1, pp. 35-52, 2004.

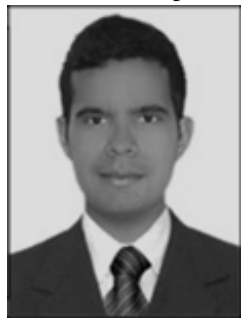

A.M. Paredes-Rodríguez, nacido el 25 de febrero de 1994 en San Pedro, Valle del Cauca, Colombia. Ingeniero Industrial, estudiante de Maestría en Ingeniería Industrial de la Universidad del Valle, Valle del Cauca, Colombia. Docente tiempo completo del Programa de Ingeniería Industrial de la Corporación Universitaria Minuto de Dios, Buga, Valle del Cauca. ORCID: http://orcid.org/0000-0001-9196-9965

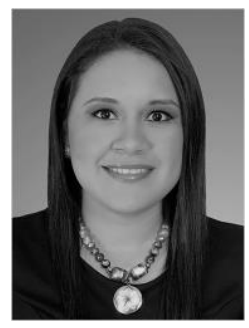

V.L. Chud-Pantoja, nacida el 3 de diciembre de 1988, Cali, Valle del Cauca, Colombia. Ingeniera Industrial, Magister en Ingeniería Industrial de la Universidad del Valle, Santiago de Cali, Valle del Cauca, Colombia. Actualmente Profesora asistente del área de Ingeniería Industrial en la Universidad del Valle Sede Zarzal.

ORCID: http://orcid.org/0000-0001-8842-637X

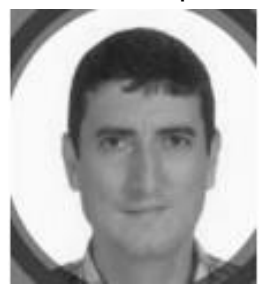

J.C. Osorio-Gómez, nacido el 20 de septiembre de 1975 en Buga, Valle del Cauca, Colombia. Ingeniero Industrial, Especialista en Logística, Magister en Ingeniería Industrial, Doctor en Ingeniería Industrial de la Universidad del Valle, Santiago de Cali, Valle del Cauca, Colombia. Actualmente Profesor titular de la Escuela de Ingeniería Industrial de la Universidad del Valle. ORCID: http://orcid.org/0000-0001-5625-5609 management system," Proc. 2013 Int. Conf. Ind. Eng. Syst. Manag., 